\title{
Elementos para uma reflexão sociológica sobre o fenômeno da mobilidade de investigadores e cientistas
}

\author{
Sandra Moreira' \\ Emília Rodrigues Araújo²
}

\section{Resumo}

O presente texto dedica-se à discussão do conceito de mobilidade de profissionais altamente qualificados e sua relação com os fenômenos de fuga e circulação do conhecimento. Num contexto de declínio demográfico e consequente reforço da mobilidade de estudantes, investigadores e cientistas, assim como outros profissionais, muitos países colocam em prática medidas para atrair novos talentos que possam reforçar as dinâmicas das suas instituições e apoiar o seu desenvolvimento econômico. As conclusões a que chegamos mostram a necessidade de repensar a mobilidade destes profissionais a partir de um quadro analítico que considere os modelos e os processos de estratificação da ciência no mundo.

Palavras-chave: mobilidade, conhecimento, circulação.

\section{Introdução}

Este texto constitui uma reflexão sobre o conceito de mobili-
dade de profissionais altamente qualificados, nomeadamente

1 Mestre em Políticas Comunitárias e Desenvolvimento Territorial, desenvolve funções no gabinete de relações Internacionais da Universidade do Minho.

2 Professora doutora do Departamento de Sociologia da Universidade do Minho. Tem pesquisas no âmbito da sociologia do tempo e estudos sociais de ciência e tecnologia. Email: emiliararaujo@gmail.com. 
investigadores e cientistas. A mobilidade de cientistas e de investigadores surge nas últimas décadas como um importante motor de transmissão de conhecimento e inovação e, por conseguinte, de desenvolvimento, a vários níveis (ACKERS, 2005; ARAÚJO, 2007; DELICADO, 2008; SOLIMANO, 2008). Este processo pode ser definido como o movimento geográfico de investigadores entre países. Por norma, considera-se que esta mobilidade tem como objetivo o desenvolvimento das suas competências, acedendo a melhores oportunidades na carreira acadêmica científica (MAHROUM, 2000; ACKERS, 2005; PIRRALHA, FONTES; ASSIS, 2009: 3). Neste contexto, e nos tempos atuais, abraçar a carreira de investigador e cientista corresponde, globalmente, a assumir o compromisso de investir na realização de percursos de mobilidade geográfica internacional (MEYER et al., 2001). Este imperativo surge a partir da constatação dos efeitos da crise econômica e financeira sobre os sistemas de ciência e tecnologia, assim como da intensificação dos mecanismos de avaliação de desempenho que contam, cada vez mais, com a internacionalização como indicador de desempenho. Múltiplos fatores podem ser cruzados, na tentativa de descortinar como se adaptam os indivíduos a este condicionamento e de que forma a mobilidade influi sobre a capacidade de a ciência e a tecnologia contribuirem para a melhoria das condições de equilíbrio e de justiça no mundo. Múltiplos são também os pontos de vista sobre os mecanismos e os efeitos que as mobilidades desencadeiam para os países e sociedades envolvidas. Uns tendem a enfatizar a mobilidade como um percurso natural essencial à economia do conhecimento. Outros, olham para esta realidade como resultado de roturas sistemáticas nos modelos políticos e econômicos, nomeadamente em contexto de erosão das fronteiras dos estados-nação. Com efeito, no caso da Europa, existem estudos (COMISSÃO EUROPEIA, 2008) observando vários obstáculos à manutenção e fortalecimento do estoque regional de investigadores qualificados necessários para alimentar o sistema de investigação e inovação europeu (COMISSÃO EUROPEIA, 2008; SOLIMANO, 2008). Entre eles, constam a dificuldade de atração e a frágil capacidade de retenção de investigadores. É certo que a crescente internacionalização da ciência conduziu a uma maior 
mobilidade dos cientistas, o que tornou as carreiras científicas mais globais (DELICADO, 2008).

É certo que nos últimos anos se tem assistido, fruto do progresso das abordagens neoliberais, a uma forte intensificação do discurso que incentiva á mobilidade e a posiciona como elemento fundamental na avaliação das carreiras científicas e das unidades de investigação. Tais discursos têm enfatizado as qualidades da profusão da sociedade de conhecimento, através do incentivo à valorização da mobilidade de profissionais altamente qualificados como um elemento básico à circulação do conhecimento, num mundo global. De qualquer forma, esses discursos carecem de problematização, designadamente quando se atravessa na Europa um estado de crise econômica endêmica e quando se assiste à emergência de um outro mapa geocientífico, marcado por novas configurações estratégicas no mundo, com a entrada na corrida mundial pelo talento de países até então não ou menos considerados, como a China e a Índia (SOLIMANO, 2008:10). O entendimento da mobilidade de investigadores e de cientistas nas óticas das abordagens sobre a "fuga de cérebros", o "ganho de cérebros" (brain gain) e o "desperdício de cérebros" (brain waste) são, assim, cruciais para a melhoria da efetividade política no campo da ciência e tecnologia, mas também da inovação e da qualificação populacional.

Tal como percebemos, a temática da mobilidade internacional de investigadores é extensa e, desde logo, bastante explicada pelas ondas e pelos ciclos de prestígio e de excelência que vão marcando as subculturas científicas e também as prioridades políticas e as formas de avaliação de desempenho individual e institucional/ coletivo. $\mathrm{O}$ fato de ser pulverizada de elementos intangíveis faz com que seja necessário redobrar o cuidado na formulação de políticas tendentes a dinamizar a mobilidade de profissionais altamente qualificados ligados à investigação e à ciência, desde logo porque o investigador não se configura apenas na sua definição como célula de capital humano. Tem uma vida carregada de sentidos e sedimentada em trajetórias espaço-temporalmente condicionadas ${ }^{3}$.

3 As análises de David Harvey e Massey a propósito do espaço e do tempo e capitalismo são extremamente relevantes e inspiradoras neste contexto. 
Elementos para uma reflexão sociológica sobre o fenômeno da mobilidade...

Sandra Moreira • Emillia Rodrigues Araújo

O objetivo do presente texto consiste, assim, em debater os principais argumentos que se colocam às abordagens sobre a mobilidade de investigadores, na perspectiva de contribuir para a consolidação de algumas ideias sobre as melhores práticas políticas no que concerne à gestão equilibrada de competências no espaço nacional e global. Deste modo, encontra-se dividido em duas pontes centrais. Num primeiro ponto concentramo-nos na explicitação histórica do conceito de mobilidade, relacionando-o com a evolução das abordagens sobre o conhecimento como bem comercializável transferível. Neste ponto, procuramos salientar os argumentos que justificam ainda hoje o retorno à análise sobre os fenômenos de "fuga de cérebros", sempre que estão em causa relações assimétricas entre países de destino e países de origem dos investigadores. No segundo ponto debruçamo-nos sobre a relação entre mobilidade e conhecimento, mostrando as tendências atuais na gestão do conhecimento que favorecem uma cultura de mobilidade.

\section{A mobilidade de investigadores e cientistas e a permanência da "fuga de cérebros"}

Tal como vários autores têm demonstrado (BRANDI, 2001; SOLIMANO, 2008), uma das linhas teóricas mais recentemente desenvolvida pelas teorias sobre a mobilidade científica assenta no pressuposto de que a troca, o intercâmbio e a mobilidade de investigadores produzem efeitos positivos para todas as partes envolvidas (PEIXOTO, 2001; GÓIS; MARQUES, 2007). Este pressuposto baseia-se na definição de conhecimento como algo compreendendo elementos intangíveis, tais como a tecnologia, a criatividade e a inovação que, por sua vez, integram um processo contínuo de aquisição, incorporação e transformação (WESTERN, 2008). Assenta, ainda, na valorização do indivíduo como eixo de capital humano (STRAUBHAAR, 2000: 17; BOZEMAN, DIETZ; GAUGHAN, 2001; SOLIMANO, 2008: 1; BENTO, COTTA; ARAÚJO, 2009). De modo 
sintético, a mobilidade científica é definida como o movimento dinâmico de investigadores entre países, através do qual se pode pensar em termos de circulação do conhecimento e de internacionalização. No fundo, tal como proposto por Williams (2006), considera-se que o indivíduo é um portador de conhecimento e tem capacidade de o transferir a outros, daí criando redes científicas e sociais (DIETZ, 2004; BOZEMAN, DIETZ; GAUGHAN, 1999: 5-6; HAKALA, 1998) relevantes para a inovação e a competitividade. A mesma abordagem tem a particularidade de sustentar-se na hipótese de que a mobilidade de talentos não é unidirecional mas multidirecional, uma vez que conduz a uma partilha de benefícios entre países de envio e de acolhimento (BRANDI, 2001; SOLIMANO, 2008:1, COTTA; BENTO; ARAÚJO, 2009; KURKA, 2007: 493). Além disso, propõe que os profissionais com sucesso no exterior contribuem, em grande parte dos casos, para o desenvolvimento dos seus próprios países, porque constituem uma almofada para o surgimento de diásporas científicas (BRANDI, 2001; KURKA, 2007; DELICADO, 2008). Em síntese, sobre esta ótica, seria antecipável reivindicar, principalmente, os efeitos positivos para os sistemas científicos nacionais (países de origem dos profissionais) decorrentes da contratação/aceitação de investigadores estrangeiros. Seria de considerar, ainda, a ausência de pressupostos deterministas na análise da mobilidade, sendo os seus efeitos resultantes dos modelos de gestão de recursos humanos nas respectivas unidades de investigação.

Assistimos hoje a um discurso tendencialmente assertivo sobre as condições objetivas da Europa para potenciar a concentração de capital humano e manter a centralidade nos domínios da inovação científica e tecnológica (COMISSÃO EUROPEIA, 2008; SOLIMANO, 2008). Contudo, este posicionamento político é sustentado pela grande incerteza relativamente ao cumprimento desse desígnio. Com efeito, os autores (GAILLARD; GAILLARD, 1998: 107; ACKERS, 2005; SOLIMANO, 2008) têm notado que uma preocupação central em vários países da Europa, designadamente dos que se consideram historicamente dominantes e atrativos, do 
ponto de vista científico e tecnológico, é o destino da comunidade científica nacional se os seus melhores graduados continuarem a emigrar em busca de melhores condições e infraestruturas para o desenvolvimento da atividade de investigação. Entre outras, esta preocupação confere valor às teses sobre a persistência de processos de "fuga de cérebros" nas sociedades modernas, não obstante a importância crescente das análises que tendem a perspectivar a mobilidade como um eixo de reforço dos mecanismos de desenvolvimento da sociedade de conhecimento (SOLIMANO, 2008).

Solimano (2008:1-2) esclarece que os debates sobre a mobilidade de profissionais altamente qualificados encetados nas décadas de 1960 e 70 do século passado eram dominados por duas perspectivas: a nacionalista e a internacionalista. No entender de Solimano (2008:2-3), ambas as abordagens conduziram a resultados distintos no que respeita à análise do impacto da "fuga de cérebros" para o crescimento econômico (RIZVI, 2005: 182). Ainda segundo o autor (SOLIMANO, 2008: 1-3), a escola "nacionalista" (Patinkin, 1968), salientava as assimetrias entre os países de origem e os de acolhimento, no que respeita à distribuição dos ganhos resultantes da mobilidade internacional de recursos humanos qualificados. Por seu turno, a tese "internacionalista" (JOHNSON, 1968) perspectivava a mobilidade de trabalhadores qualificados como uma mais-valia, pois a mobilidade de recursos humanos de locais de baixa produtividade para locais de alta produtividade resultaria "num crescimento econômico mundial e na melhoria das condições de vida no mundo" (SOLIMANO, 2008: 1-2).

Os autores que se debruçam sobre a história do conceito e das ideologias que o fundamentam, tal como Brandi $(2001 ; 2004)$ e Solimano (2008) dão conta de que a "fuga de cérebros" era discutida, na época, como designativo de um fluxo unilateral de mobilidade de profissionais altamente qualificados, ou seja, como uma movimentação unidirecional de capital humano. Sugere-se que a fragilização progressiva do colonialismo permitiu a emergência de uma classe intelectual nos novos países independentes, a qual, devido à falta de infraestruturas académicas, se movimentou para 
novos destinos com melhores condições para o desenvolvimento da sua atividade, como eram a Austrália, o Canadá, os Estados Unidos, a França, o Reino Unido e a Alemanha Ocidental. Nesta altura, a literatura sobre a "fuga de cérebros" passa a defini-la como a saída líquida de profissionais qualificados das ex-colônias para as antigas nações imperiais europeias e da Europa para a América do Norte, sobretudo Estados Unidos (JOHNSON, 1979: 7; BRANDI, 2001, 2004). Mas, tal como reiteram os autores (BRANDI, 2001; 2004; SOLIMANO, 2008), vários líderes mais nacionalistas consideravam a "fuga de cérebros" um fenômeno neo-colonialista, insistindo que o crescimento das suas nações estava a ser "frustrado" pela emigração dos seus talentos mais jovens ${ }^{4}$, atribuindo a este fato, inclusive, as razões do seu subdesenvolvimento (RIZVI, 2005: 181; AVVEDUTO; BRANDI, 2004: 2). É efetivamente notório que os baixos salários e as deficientes condições políticas e de trabalho que caracterizavam alguns países, constituíam incentivos à saída de profissionais qualificados para nações mais desenvolvidas. Do lado da procura, os principais fatores de atração eram os melhores salários e a existência de indústrias baseadas no conhecimento, emergentes nos países mais ricos e a necessitar de mão de obra qualificada. A "fuga de cérebros" caracteriza-se, então, como um movimento dos países do hemisfério Sul (tradicionalmente menos desenvolvidos) para os países do Norte (CARRINGTON; DETRAGIACHE, 1999), o que se apresenta ser especialmente revelador de relações de dominação e dependência e, em alguns casos, de exploração de uns países sobre outros. Segundo Tesón (2008) a "fuga de cérebros" explica-se por duas leis econômicas bem definidas: a lei da oferta e da procura de trabalho e a lei das vantagens comparativas. Importa perceber, tal como demonstram Brandi (2001) e Solimano (2008) que a abordagem predominante em fase pós-imperialista acentuava uma concepção da mobilidade como propícia à "fuga de cérebros" e, portanto, susceptível de

4 Esta fuga, à qual se ligam fatores políticos e ideológicos é bem marcante hoje na Venezuela (MARGOLIS, 2009). 


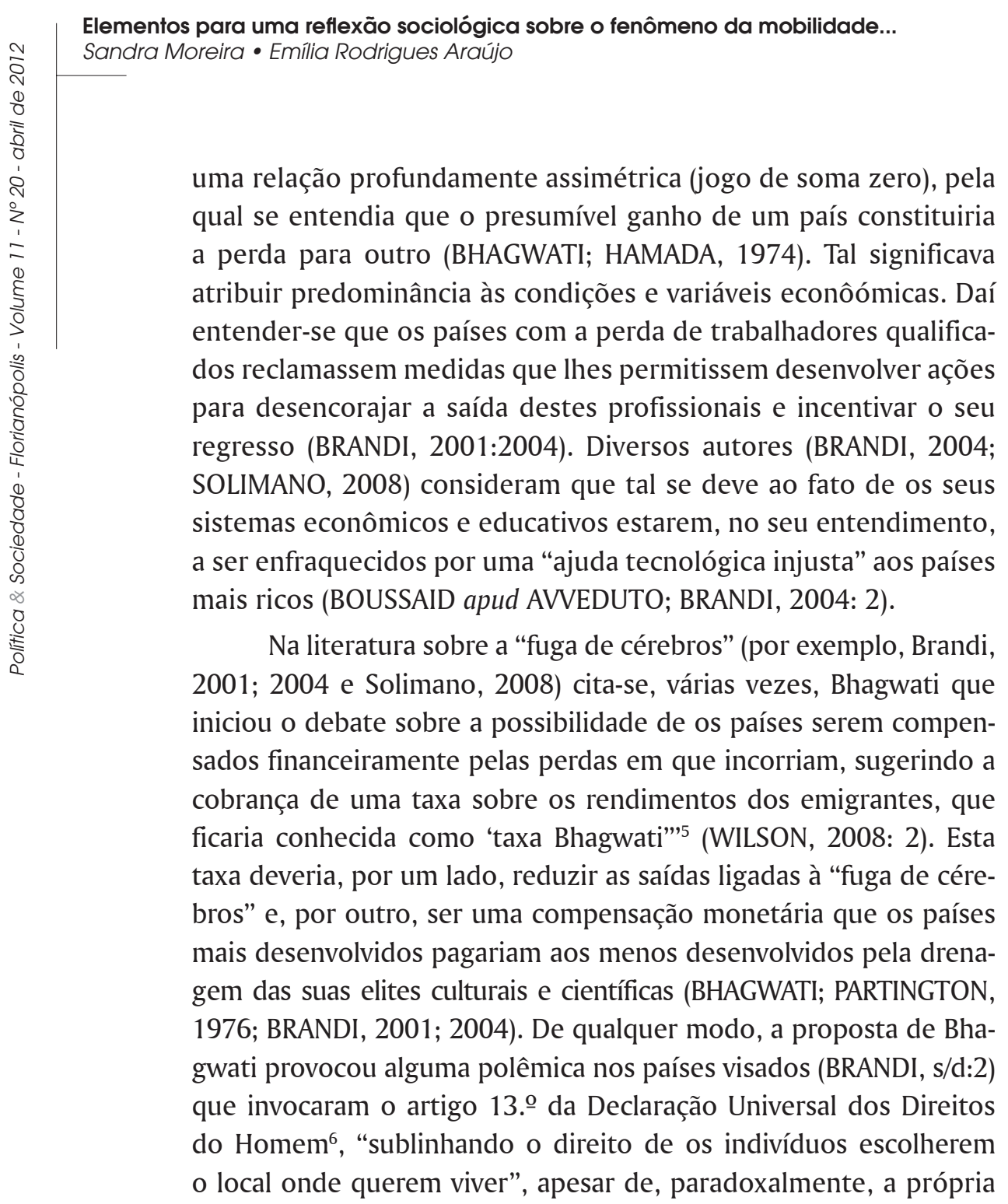

5 A proposta de Jagdish Bhagwati, consistia em aplicar uma taxa sobre os rendimentos dos profissionais emigrantes nos países desenvolvidos em favorecimento dos países em desenvolvimento. Esta taxa seria cobrada sob os auspícios das Nações Unidas com a ajuda das autoridades fiscais dos países desenvolvidos.

6 Declaração Universal dos Direitos do Homem - Artigo 13\%:

1. Toda a pessoa tem o direito de livremente circular e escolher a sua residência no interior de um Estado.

2. Toda a pessoa tem o direito de abandonar o país em que se encontra, incluindo o seu, e o direito de regressar ao seu país. 
mobilidade poder ser provocada pelo fato de os países de origem não responderem às necessidades dos seus cidadãos, "o que constituiria também uma matéria discutível no âmbito dos direitos humanos" (BRANDI, s/d:2). O certo é que aquela interpretação está na origem das diversas medidas tendentes a restringir a presença dos profissionais nos países de recepção e que são conhecidas em alguns países atualmente, como o Brasil e a Austrália.

Brandi, citando Boulier (1999) refere que em 1978, um estudo do Instituto das Nações Unidas para a formação e a pesquisa (UNITAR) veio "demonstrar que muitos dos emigrantes, designadamente os mais qualificados, regressavam ao seu país de origem numa fase mais avançada da carreira, acabando por desempenhar um papel importante no seu desenvolvimento científico" (BRANDI, $\mathrm{s} / \mathrm{d}: 2)$. Os autores revelam ser por esta altura que se inicia um debate promovido pelos defensores da tese internacionalista sobre a existência de um fluxo exagerado de cérebros (brain overflow), e não propriamente de "fuga de cérebros". Este novo conceito aplicava-se a situações em que os países de origem detinham (intencional ou não intencionalmente) um excesso de profissionais qualificados, cujo mercado de trabalho interno não conseguia absorver (BALDWIN, 1970; FIHEL et al., 2007: 6)

Tal como esclarecem os autores (BRANDI, 2001, 2004; GIANNOCCOLO, s/d:3), o aparecimento da chamada nova teoria do crescimento $^{7}$ (CORTRIGHT, 2001), assente nos trabalhos de Romer e Lucas, fez emergir uma nova ênfase sobre o papel desempenhado pelo capital humano como fator de produção e no aumento dos retornos do conhecimento, ou capital humano acumulado (CARRINGTON; DETRAGIACHE, 1999). Esta tese veio sedimentar a ideia de que a migração de profissionais qualificados potencia efeitos tecnológicos

7 A nova teoria do crescimento também designada de teoria do crescimento endógeno, é uma perspectiva da economia que encara o progresso tecnológico como um produto da actividade económica. Entende que, apesar de não serem objectos físicos, o conhecimento e a tecnologia são caracterizados por grandes retornos, conduzindo ao crescimento (CORTRIGHT, 2001:2). 
positivos para as economias de acolhimento, uma vez que aumenta a disponibilidade de capital humano. Além disso, entende-se que os trabalhadores mais qualificados permitem baixar os custos de produção dos países e aumentar a qualidade, tornando-os mais competitivos (BRANDI, 2001, 2004; ACKERS, 2005; SOLIMANO, 2008). Tal como faz ver Brandi (s/d: 2-3), no decorrer dos anos de 1990, um outro aspecto relevante é a mobilidade de profissionais para economias emergentes, cuja abertura se explica com base nas profundas alterações políticas e económicas vividas em países como a Polônia e outros do Sudeste Asiático (AVVEDUTO; BRANDI, 2004: 3).

Tal como documenta a literatura (GIANNOCCOLO, 2006), os sucessivos estudos realizados pela OCDE deram conta, ao longo da década de noventa, do número elevado de saídas de cientistas dos países de Leste em transição para os EUA e outros países da europa central (DE TINGUY e de WENDEN, 1993 apud BREINBAUER, 2007; SHKOLNIKOV, 1995: 103-105). Vários autores citados por Giannoccolo (2006), sustentam que esta corrente migratória de Leste constituiu uma das maiores evidências de "fuga de cérebros", sendo que um número significativo daqueles profissionais teve sérias dificuldades de integração, acabando por se tornar vítima do chamado "desperdício de cérebros" (brain waste), em particular em países como a Alemanha e o Reino Unido, por não encontrar empregos nos países de acolhimento apropriados ao seu nível oficial de qualificações. Com efeito, este termo foi utilizado por alguns autores para evidenciar as dificuldades de inserção profissional e social, aliadas a conflitos de ordem intercultural ${ }^{8}$. Conforme foi dito anteriormente, até início dos anos de 1990, a "fuga de cérebros" constituía o conceito predominante (ainda que controverso) nas teses sobre a saída de recursos humanos qualificados do seu país de origem com destino às economias industrializadas. Agora juntava-se-lhe o conceito de "desperdício de cérebros" que traduzia o subaproveitamento de

8 Países em transição ou economias em transição: países que passaram de uma economia orientada centralmente para uma economia de mercado. Nesta categoria inserem-se países como a China, Mongólia, Vietname, as antigas repúblicas soviéticas e os países da Europa Central e Oriental (SOUBBOTINA, 2004). 
qualificações os países de repceção, daí que durante este período se tenham desenvolvido abordagens consistentes com a ideia sobre a existência de desigualdade nas relações de dependência entre os países desenvolvidos e os países em desenvolvimento, mais tarde retomadas por autores como Immanuel Wallerstein que, na mesma linha de Stephan Castells e Raul Wise, encara a "fuga de cérebros" como uma consequência da estrutura do mundo capitalista, que cria condições de crescimento econômico para alguns países e de subdesenvolvimento para outros, dada a aplicação de diferentes formas de controle de trabalho e de distribuição do poder político que assenta numa divisão internacional do trabalho que determina as relações entre diferentes regiões, bem como as condições de trabalho em cada região.

Tal como afirma Brandi (s/d:2), os anos de 1980 trouxeram importantes inovações ao nível dos meios de comunicação, designadamente o uso da internet e a constituição de redes de informação, permitindo a internacionalização da investigação sem necessidade de uma deslocação geográfica dos investigadores. Tais ferramentas impulsionaram, igualmente, uma maior aproximação e interação entre os profissionais que emigravam e os que permaneciam nos países de origem. Assim, esta década e a seguinte (anos noventa) ficaram marcadas, em contexto europeu, pela promoção da mobilidade e do intercâmbio de conhecimento, uma realidade constituída através do lançamento de várias iniciativas formais por parte da União Europeia, como o programa Socrates/Erasmus, criado em 1987. Só no primeiro ano (1987/88), participaram neste esquema de mobilidade mais de 3.200 alunos europeus ${ }^{9}$. Verifica-se também uma intensificação dos movimentos migratórios resultantes da criação do Espaço Shengen em 1985, e da implementação do Tratado de Maastricht, após 1992, permitindo a abolição das fronteiras físicas e a livre circulação de pessoas dentro do espaço europeu. Os autores identificam um novo impulso para a mobilidade de profissionais altamente qualificados de-

9 Ver o site oficial em: <http://ec.europa.eu/education/erasmus/doc/stat/table1.pdf >. 
corre, paralelamente, de uma política de expansão das empresas multinacionais, bem como do fomento de programas de ajuda internacional desenvolvidos, sobretudo, por organizações de caráter humanitário (BRANDI, 2001, 2004; SOLIMANO, 2008).

Conclui-se que se a perda de recursos humanos qualificados para o país de origem revestia o conceito de "fuga de cérebros" de uma conotação negativa e pejorativa, a década de noventa trouxe uma releitura do fenômeno entre os teóricos, que tentam salientar os aspectos positivos do mesmo imprimindo, assim, uma mudança de paradigma, através da qual se fazem ver os benefícios para os países de origem (ganho de conhecimento) decorrentes da mobilidade (BRANDI, 2001; SOLIMANO, 2008). A literatura sobre este tema, também conhecida como incerta na " nova economia da fuga de cérebros" (STARK, 2005), refere que a saída de capital humano comporta benefícios, tais como o envio de remessas, favorecendo o crescimento econômico do país de origem e um possível investimento em educação (SOLIMANO, 2008:3). Além disso, a possibilidade de emigrar e auferir de um melhor salário no estrangeiro cria incentivos para os indivíduos aumentarem os seus níveis educativos, permitindo a criação de um estoque de capital humano, pois nem todos conseguem atingir o desígnio de emigrar (DOCQUIER; SCHIFF, 2009: 29; SOLIMANO, 2008:3). A criação de diásporas é outra das vantagens apontadas, dado que potenciam, muitas vezes, a transferência de conhecimento e tecnologia a um custo relativamente baixo, mediante a constituição formal ou informal de redes e com retornos importantes para os países de envio (LOWELL; FINDLAY; STEWART, 2004: 22; SOLIMANO, 2008:3). Os efeitos positivos da diáspora poderão consistir, igualmente, na transferência de normas ou no incentivo a reformas institucionais, afetando indiretamente a promoção do desenvolvimento econômico naqueles países (BRANDI, 2001; DELICADO, 2007; SOLIMANO, 2008; MARCHIONI et al., 2009: 5). A atração de investimento direto estrangeiro, através do papel facilitador desempenhado pelos migrantes nas transferências entre os países de acolhimento e origem, representa também uma mais-valia (BRANDI, 2001, Ali et al., 
2007: 5). Em última análise, o ganho de cérebros verifica-se quando os profissionais qualificados regressam ao seu país, munidos de conhecimentos específicos, do gosto pela inovação e de uma rede privilegiada de contatos (LOWELL; FINDLAY; STEWART, 2004: 20; FONTES, 2007; DELICADO, 2008; SOLIMANO, 2008). Por isso, diversos autores (BRANDI, 2001; DOCQUIER E RAPOPORT, 2004:3; FONTES, 2007; DELICADO, 2008, entre outros) consideram os emigrantes qualificados continuam a contribuir para a economia do país de origem, pois, tal como sintetiza Solimano contribuem com remessas, qualificações e redes de relacionamento favoráveis à melhoria do comércio e difusão do conhecimento (2008: 3-4).

A ideia de que os países em desenvolvimento, usualmente mais afetados pela "fuga de cérebros", poderiam obter ganhos através da mobilidade dos seus profissionais altamente qualificados alcançou consistência com o processo de globalização (ACKERS, 2005; SOLIMANO, 2008) que se caracteriza pela mobilidade internacional de pessoas, bens, capitais e tecnologia, sugerindo, $a$ priori, uma maior partilha dos benefícios produzidos. Esta nova abordagem veio sublinhar a necessidade de alterar o discurso vigente, assente na dicotomia entre "fuga de cérebros" e "ganho de cérebros", para a criação de um discurso assente numa visão mais ampla e complexa, denominada por "circulação do conhecimento" (KURKA, 2007: 493), acreditando-se que tais mobilidades possuirão uma natureza circulatória e um caráter temporário (ACKERS, 2005; SOLIMANO, 2008). Quer dizer, defendendo as vantagens dos movimentos de cientistas e investigadores de um lugar para outro, facilitadas pelas possibilidades de difusão do conhecimento (CASEY, et al. 2001: 13 apud BREINBAUER, 2007)

O surgimento da tese sobre a circulação de conhecimento (brain circulation) veio alterar, fundamentalmente, o modo de conceber as oportunidades de desenvolvimento das "periferias", advogando-se que os profissionais com sucesso no exterior pretendem contribuir para o desenvolvimento dos seus próprios países e fazem-no (SOLIMANO, 2008). Um exemplo apontado por vários autores é o do impacto transformativo experienciado pela China e 
pela Índia através da ligação e troca de informação com os seus investigadores sediados em Silicon Valley (SOLIMANO, 2008: 4). Este autor (SOLIMANO, 2008) descreve que a ligação destes com os países de origem é feita através do desenvolvimento de operações em nível local, da criação de empresas, do investimento em empresas novas ou já existentes, da construção de parcerias inter-regionais, entre outras. Estes profissionais migrantes colaboram com o poder político e a indústria para identificar e remover os obstáculos ao desenvolvimento local (SAXENIAN, 2002). O estabelecimento de redes transnacionais é, pois, considerado essencial para a manutenção da circulação de cérebros, contribuindo para o desenvolvimento econômico dos países emissores (SMITH; FAVELL, 2006). A globalização permitiu, ainda segundo os autores mencionados, criar um mercado de trabalho à escala global. $\mathrm{O}$ surgimento de novas oportunidades nos países recentemente industrializados ajudou, também, no reforço da migração dos trabalhadores qualificados dos países desenvolvidos para os países em desenvolvimento (BRANDI, 2004: 12). Mas, ao mesmo tempo, como frisam os autores (ACKERS, 2005; SOLIMANO, 2008) as economias mais industrializadas (Estados Unidos, Canadá, Austrália, França, Reino Unido, entre outros) passaram a competir entre si pela atração de pessoal técnico e científico altamente especializado (SMITH; FAVELL, 2006), assumindo-se como líderes na construção e validação de rankings institucionais altamente favoráveis à concentração de recursos humanos, materiais e simbólicos.

Com efeito, o passar dos anos veio demonstrar que os países menos desenvolvidos continuam a sofrer as maiores perdas numa competição por talentos olhada por cada vez mais países como "injusta”, no contexto da globalização. Lembremos o que nos diz Martine (2005:11) "no curto prazo, a migração pode servir como "válvula de escape", aliviando as pressões sobre o mercado de trabalho e trazendo o dinheiro muito necessitado para as famílias. No longo prazo, a perda de trabalhadores mais qualificados, assim como da população jovem, e a dependência sobre as remessas podem constituir obstáculos para o desenvolvimento (PELLEGRINO, 
2003: 27) ". Desde 1999 que os dados de vários estudos (CARRINGTON; DETRAGIACHE, 1999; BANCO MUNDIAL e OCDE) têm confirmado a existência de uma "fuga de cérebros" substancial para os Estados Unidos, particularmente de países de África, América Central, e Caraíbas. Estudos citados, como os de Docquier e Marfouk (2004), indicam que os países da OCDE concentram mais de $80 \%$ dos imigrantes qualificados, apesar das diferenças internas. As perdas assinaláveis dos países mais pobres e com menos recursos e, em simultâneo, a elevada dependência dos que regressam face às possibilidades de emprego promovidas pelos países, são justificações fortes para ser retomado o debate sobre a "fuga de cérebros" na atualidade (VINOKUR, 2006) ${ }^{10}$. Como já salientamos, aliás, a crise econômica na Europa está a dar agora sinais claros de novas intensas vagas de saída de profissionais qualificados, entre os quais investigadores e cientistas rumo a países europeus centrais (Alemanha e Reino Unido), mas também os Estados Unidos e o Canadá.

\section{A mobilidade e o conhecimento}

Em face das mudanças constantes no sentido da globalização, é relevante tratar, em específico, o conceito de mobilidade, articulando-o com o de produção e transferência de conhecimento, pois é este processo que mais relevância tem nas teorias recentes, atendendo à tipologia dos países envolvidos, áreas científicas e fatores culturais estruturantes nas práticas de investigação e inovação. O conhecimento é hoje entendido como um fator primordial de crescimento econômico. Aspectos intangíveis como a tecnologia, a criatividade e a inovação integram um processo indutor de crescimento que tem na sua base o capital humano ou a capacidade dos indivíduos para desenvolverem ideias e objetos (SOLIMANO, 2008: 1). Por isso, tem-se tornado cada vez mais importante investigar

10 A "fuga de cérebros" foi central na Conferência Ministerial Euro-Africana sobre Migrações e Desenvolvimento (Público, 12.06.2006) 
como o conceito de conhecimento, enquanto fator de produção, se desenvolveu comparado com outros fatores como o capital físico, o trabalho e as matérias-primas (SOLIMANO, 2008). Westeren (2008: 139) sustenta que a teoria tradicional do crescimento macroeconômico conferia reduzida ênfase à análise da criação de conhecimento até 1990, altura em que Romer introduziu o conceito de crescimento endógeno, baseado no princípio da circulação e transformação de ideias, como fonte primária da inovação.

Williams (2006) afirma que todo o migrante é um portador de conhecimento potencialmente transferível a todos com quem se relaciona. Nesta linha, a mobilidade internacional de pessoas é considerada uma das vias mais importantes para a criação e transferência de conhecimento, sobretudo porque ultrapassa fronteiras. Os migrantes, particularmente os mais qualificados, incorporam consigo um conhecimento que, articulado com aquele que adquirem pela sua participação em práticas formais e informais, leva à criação, transformação e expansão de conhecimento dentro e fora das organizações ou países. Há cada vez mais o reconhecimento de que os indivíduos móveis têm potencial para criar redes científicas e sociais translocais contribuindo decisivamente para a inovação e competitividade. As redes sociais que se criam num determinado domínio do saber não só favorecem o aumento da produção científica como servem de recurso para oportunidades de emprego e mobilidade, e revelam aplicações para produtos de trabalho científico e técnico (FONTES et al., 2011). Estas redes, a que Diana Crane chama invisible colleges (ÁVILA, 1997: 17) são construídas através de relações interpessoais que facilitam diversas formas de comunicação e colaboração entre grupos de cientistas e que permitem a troca de ideias numa área comum de investigação ou em áreas afins (DIETZ, 2004).

Importa, para o presente texto, determo-nos na importância da distribuição ou transferência de conhecimento que, segundo o mesmo Westeren (2008), é definida por vários autores como “o processo através do qual uma unidade (grupo, departamento, divisão) é afetada pela experiência de outra". O desenvolvimento 
exponencial das tecnologias de informação e comunicação criou uma maior necessidade de técnicos e especialistas em diversas áreas, tornando o talento humano particularmente valioso (SOLIMANO, 2008). De resto, o uso das competências é um pré-requisito para a criação de novo conhecimento. A dimensão nacional do capital humano mudou radicalmente nos últimos anos. No campo da ciência, os resultados da globalização incluem a integração da investigação, o uso do inglês como língua franca nas comunicações científicas, o crescente mercado de trabalho internacional para acadêmicos e cientistas, o aumento das empresas de comunicação e das publicações multinacionais (ALTBACH; KNIGHT, 2007: 291). Nos dias de hoje, a internacionalização parece ser de tal forma uma parte integrante da investigação que os cientistas raramente mencionam razões específicas para a sua existência, apenas a consideram ser uma condição sine qua non (HAKALA, 1998). Os avanços tecnológicos e os modernos sistemas de telecomunicações e transportes fizeram também reduzir os custos das transações e dos movimentos de capital humano que se tornou-se internacionalmente móvel. As pessoas altamente qualificadas têm agora a oportunidade de comunicar e vender o seu conhecimento em todo o globo (STRAUBHAAR, 2000: 17), sem necessidade de se deslocarem (BRANDI, 2001; 2004). Torna-se, aliás, muito fácil que um estado sofra drenagem considerável dos seus cérebros, mesmo sem saber ou sem os contabilizar.

De qualquer forma, as tecnologias não resolvem nem servem todos os interesses e processos. Apesar de hoje se poder afirmar que o processo de transferência de conhecimentos se dá independentemente da presença física do indivíduo, a transmissão do conhecimento tácito implica proximidade física (social e cognitiva), de modo a tornar-se eficiente. Williams (2006) defende que a transmissão do conhecimento incorporado (embodied knowledge), ou seja, o conhecimento resultante das experiências vividas através da presença física, só é possível mediante a mobilidade física (corpórea) dos indivíduos. O mesmo se passa com o conhecimento embebido (embedded knowledge), ou o conhecimento adquirido através de fatores contextuais. De fato, enquanto o conhecimento 
explícito pode ser transmitido por meios formais e sistematizados (manuais, bases de dados etc.), o conhecimento tácito existe apenas nas pessoas e nos contextos de ação. Daí que a melhor forma de o transmitir seja através da mobilidade física.

Perante o exposto, podemos concluir que a mobilidade constitui uma forma de os investigadores alcançarem e gerarem reconhecimento através da sua integração em centros e equipas que se procura serem de "excelência", dando-se, assim, relevo às suas condições de atração de recursos materiais, humanos e simbólicos. Embora possam existir posições a favor e contra a sua concretização, dependendo dos benefícios que dela resultam, parece ser entendimento comum no campo da ciência que sem mobilidade não existe circulação de conhecimento, inovação e desenvolvimento ${ }^{11}$. Esta posição tem ganho relevância nos últimos anos, particularmente no contexto europeu.

\section{Conclusão}

O tema da mobilidade de investigadores e de profissionais altamente qualificados tem vindo a ganhar relevância social, política e sociológica. Segundo um modelo de entendimento neo-liberal, a mobilidade de investigadores e de cientistas representa, fundamentalmente, a mobilidade de capitais científicos. Por isso, ela não é só necessária como inevitável num mundo global. A circulação do conhecimento é entendida, nessa ótica, como o termo mais correto para definir esse processo de circulação de indivíduos e dos respetivos capitais. Todavia, levando em conta as roturas introduzidas pela vulnerabilidade do capitalismo financeiro, torna-se cada vez mais pertinente retomar algumas problemáticas que constituíram debate nos anos cinquenta do século passado, em

11 Ávila afirma, a respeito de Merton, que este autor "refere que, no geral, se assiste na instituição científica a um efeito de sobrevalorização das contribuições dos cientistas que já adquiriram uma reputação científica considerável e, inversamente, a um efeito de subvalorização do desempenho daqueles que ainda não foram reconhecidos" (ÁVILA, 1997: 11). 
particular as que liam na mobilidade de cientistas e de investigadores, assim como de outros profissionais, como um processo de perda significativa de valor e de capital para os países de origem, os quais se consideravam estar em situação de domínio por parte de países historicamente mais centrais, no plano Europeu e mundial.

Ao longo deste trabalho, propusemo-nos problematizar os conceitos de mobilidade e conhecimento à luz das várias correntes teóricas e das diferentes perspectivas apresentadas por países de origem e de destino de profissionais altamente qualificados. Tal como proposto por diversos autores (SOLIMANO, 2008:1-3), à visão nacionalista de que insistia no desequilíbrio de ganhos resultantes da mobilidade internacional de recursos humanos qualificados para países de origem e acolhimento, contrapunha-se a visão internacionalista, o qual advogava um benefício generalizado para todos os intervenientes no processo de mobilidade. As crescentes dificuldades econômicas evidenciadas por alguns dos países autodesignados 'vítimas' da "fuga de cérebros" fizeram centrar o debate nas perdas sofridas com a saída contínua de capital humano para economias mais fortes e competitivas (BRANDI, 2001; ACKERS, 2005; SOLIMANO, 2008).

A criação de diásporas científicas e o retorno obtido (remessas) pelos países de envio, através de uma maior prosperidade dos seus profissionais nos países de acolhimento, propiciaram que novos argumentos alterassem a visão negativa da "fuga de cérebros", assumindo a existência, primeiro, de um "ganho de cérebros", e, posteriormente, de uma "circulação de conhecimento" (ACKERS, 2005; SOLIMANO, 2008). Este fenômeno surge, essencialmente, como um produto da globalização, cujos efeitos se estendem ao mercado de trabalho, tornando-o mais global e acessível a qualquer cidadão, independentemente da sua origem geográfica. No caso dos cientistas-investigadores, a entrada neste mercado internacional é de sobremaneira importante para o seu crescimento pessoal e desenvolvimento profissional, dadas as competências envolvidas na obtenção de prestígio e reconhecimento científico, bem como para a produção de conhecimento e inovação. Este 
movimento é também potenciado por estímulos exteriores ao indivíduo, que funcionam como importantes fatores de atração, no caso, para o território europeu, como sejam a criação do Espaço Europeu de Investigação.

O declínio demográfico na Europa e a necessidade de se tornar competitiva face aos Estados Unidos e a outras economias emergentes como a China e a Índia, instam este continente a criar políticas de atração de profissionais altamente qualificados que possam dar cumprimento aos seus desígnios de se tornar uma verdadeira sociedade do conhecimento. Verificamos, contudo, que os estímulos facilitadores da mobilidade decorrentes de uma união econômica e da abolição de fronteiras físicas, não encontram sequência na vontade dos Estados-nação para a construção de políticas convergentes à plena concretização da cidadania em nível mundial e europeu. Neste sentido, os próprios investigadores, cientistas e profissionais europeus deparam-se com dilemas profundos na concretização da sua mobilidade, pela disparidade de políticas de âmbito econômico, jurídico e administrativo entre os vários países da União Europeia. Para a compreensão dos fenômenos da mobilidade devemos considerar que o sistema científico deve ser percepcionado como um palco de competição onde os cientistas, as suas instituições e países lutam pelo seu reconhecimento individual e pelo acesso aos meios (materiais e humanos) que lhes possibilitem alcançar o prestígio entre os seus pares, o que torna a destrinça entre as vantagens e as desvantagens da mobilidade para o indivíduo e para os sistemas organizacionais e nacionais um assunto sempre aberto à discussão e dependente dos contextos socioeconômicos e políticos concretos. Numa altura em que as estatísticas insistem sobre a saída acelerada de profissionais das economias que atravessam períodos de crise intensa, como Portugal, consideramos necessário rever a relação da política tanto com a fuga de cérebros, como com a circulação do conhecimento que, por mais amplo que seja, continua a ser avaliado como responsabilidade "nacional", reportando, por isso, aos países onde efetivamente residem os profissionais. Por isso, é extremamente 
importante posicionar o debate nos contextos geográficos e políticos que fazem toda a diferença na avaliação da mobilidade.

Primeira versão recebida em 17/07/2010.

Versão final recebida em 21/02/2012.

\section{Bibliografia}

ACKERS L. Gender, Mobility and Progression on Science Careers. MOBISC Summary Report: UK, Centre for the Study of Law \& Policy in Europe, 2005

ACKERS L. Moving People and Knowledge: Scientific Mobility in the European Union . International Migration Review, v. 43, n. 5, p. 99-131, Dez. 2005. Disponível em: < http://www.liv.ac.uk/www/ ewc/docs/Migration\%20workshop/Ackers-paper03.2004.pdf > . Acesso em: 2 de Dezembro de 2009

ALTBACH, P. G. e KNIGTH J. The Internationalization of Higher Education: Motivations and Realities. Journal of Studies in International Education, v.11, p. 290-305, 2007. Disponível em: $<$ http://jsi.sagepub.com/cgi/content/abstract/11/3-4/290>. Acesso em: 19 de Fevereiro de 2010.

ARAÚJO, E. Why Portuguese Students Go Abroad to Do Their PhDs. Higher Education in Europe, v. 32, n. 4, p. 387- 397, 2007.

ÁVILA, P. A distribuição do capital científico: diversidade interna e permeabilidade externa no campo científico. Sociologia Problemas e Práticas, n. 25, p. 9-49, 1997.

AVVEDUTO S., BRANDI M. Carolina . Evolution of Theories of "brain drain" and Migration of Skilled Personnel. The "brain drain" - Emigration of Qualified Scientists, Feb. 2004, UNU-MERIT, Disponível em: <http://www.merit.unimaas.nl/braindrain/>. Acesso em: 1 de Novembro de 2009. 
Elementos para uma reflexão sociológica sobre o fenômeno da mobilidade...

Sandra Moreira • Emília Rodrigues Araújo

BALDWIN, G. B. "Brain drain" or Overflow? Foreign Affairs, v. 48, n. 2, p. 358-372, Jan. 1970.

BEINE M., DOCQUIER, F., RAPOPORT, H. "Brain drain" and LDCs' Growth: Winners and Losers, IZA DP n. 819, Jul. 2003. Disponível em: $<\mathrm{ftp} / / /$ repec.iza.org/RePEc/Discussionpaper/dp819.pdf > . Acesso em: 20 de Dezembro de 2009.

BENTO, S., COTTA, D. e ARAÚJO, E. Mobility and Research Institutes in Portugal -Conceptualizing Knowledge Circulation. Conferência Internacional Academic Mobility, Tallin, Set. 2009.

BHAGWATI J. e HAMADA, K. The "brain drain", International Integration of Markets for Professionals and Unemployment: A Theoretical Analysis. Journal of Development Economics, v. 1, p. 19-42, 1974.

BHAGWATI, J. N.e PARTINGTON, M. (ed.) Taxing the "brain drain": a proposal. Amsterdam, North Holland, 1976.

BOZEMAN, B.; DIETZ, J. S.; GAUGHAN, M. Scientific and Technical Human Capital: An Alternative Model for Research Evaluation, (draft of). American Political Science Association, Ago.2, 1999.

BRANDI, M. C. The evolution in theories of the "fuga de cérebros" and the migration of skilled personnel, s/d. Disponível em: $<$ http://www.irpps.cnr.it/it/system/files/the_evolution_study.pdf $>$. Acesso em: 20 de Novembro de 2009.

BRANDI, M. C. The evolution in theories of the "fuga de cérebros" and the migration of skilled personnel. Studi Emigrazione n. 141, v. 38, 2001.

BRANDI, M.C. Brandi M.C., La Storia del Brain drain. Studi Emigrazione, v. 31 , n. 156, 2004.

BREINBAUER, A. "Brain drain" - Brain Circulation or... What Else Happens or Should Happen to the Brains - Some Aspects of Qualified Person Mobility/Migration, FIW Working Paper, n. 4, Jun. 
2007. Disponível em: < http://www.fiw.ac.at/fileadmin/Documents/ Publikationen/Working_Paper/N_004-breinbauer.pdf $>$. Acesso em: 28 de Dezembro de 2009

BRZOZOWSKI, J. P. Brain Waste, Educational Investments and Growth in Transitional Countries, Working Paper, Cracow University of Economics, Krakow, $7^{\text {th }}$ Jun.2007. Disponível em: $<$ http://papers.ssrn.com/sol3/papers.cfm?abstract_id=991785>. Acesso em: 12 de Fevereiro de 2010.

CARRINGTON, W.e DETRAGIACHE E. How extensive is the "brain drain"? Finance \& Development, v. 36, n. 2, Jun. 1999. Disponível em: <http://www.imf.org/external/pubs/ft/fandd/1999/06/carringt. htm > . Acesso em: 20 de Novembro de 2009.

COMISSÃO EUROPEIA. Report of the ERA Expert Group: realising a single labour market for researchers, 2008. Disponível em: $<$ http://ec.europa.eu/euraxess/pdf/research_policies/era_green_ paper_eg1_lowres.pdf $>$. Acesso em: 10 Janeiro de 2010.

COMISSÃO EUROPEIA. Science Policies in the European Union, Report prepared by ETAN, Brussels, 2000. Disponível em: < http:// www.bmwf.gv.at/fileadmin/user_upload/wissenschaft/frauen/g_ wo_etan_en_200101.pdf > . Acesso em: 15 de Março de 2010.

COMISSÃO EUROPEIA. Working Document: The Thinking of Commission Services on Measures Regarding the Admission of Researchers, JAI-2003-00997-00-01-EN-TRA-00 (FR), 2003. Disponível em: < http://www.uniroma1.it/internazionale/relazioni/ conferenza_51203/proceedings/EHRART_ENG.pdf > . Acesso em: 12 de Fevereiro de 2010.

CORTRIGHT, J. New Growth Theory, Technology and Learning - A Practitioners Guide. Reviews of Economic Development, Literature and Practice, n. 4 2007. Disponível em: < http://www. eda.gov/ImageCache/EDAPublic/documents/pdfdocs/1g3lr_5f7_5 fcortright_2epdf/v1/1g3lr_5f7_5fcortright.pdf $>$. Acesso em: 2 de Fevereiro de 2010. 
Elementos para uma reflexão sociológica sobre o fenômeno da mobilidade...

Sandra Moreira • Emília Rodrigues Araújo

DELICADO, A. Inquérito aos investigadores portugueses no estrangeiro - Relatório preliminar, Instituto de Ciências Sociais da Universidade de Lisboa, Out. 2007.

DELICADO, A. Cientistas Portugueses no Estrangeiro - Fatores de mobilidade e relações de diáspora. Sociologia, Problemas e Práticas, n. 58, p. 109-129, 2008. Disponível em: < http:// sociologiapp.iscte.pt/pdfs/10100/10104.pdf $>$. Acesso em: 3 de Janeiro de 2010.

DIETZ, J. S. Scientists and Engineers in Academic Research Centres - An Examination of Career Patterns and Productivity, Georgia Institute of Technology, Fev. 2004.

DOCQUIER, F. e MARFOUK. A. Measuring the international mobility of skilled workers (1990-2000), Policy Research Working Paper, n. 3381, World Bank, Washington, D.C., USA, 1994. Disponível em: <http:// www-wds.worldbank.org/external/default/WDSContentServer/WDSP/ IB/2004/09/22/000160016_20040922150619/Rendered/PDF/wps3381. pdf $>$. Acesso em: 17 de Novembro de 2009.

DOCQUIER, F., RAPOPORT, H. Skilled Migration: the perspective of developing countries, Policy Research Working Paper, World Bank, 2004. Disponível em: <http://wwwwds.worldbank.org/ servlet/WDSContentServer/WDSP/IB/2004/09/22/000160016_20 040922151739/Rendered/PDF/WPS3382.pdf> . Acesso em: 30 de Novembro de 2009.

FIHEL, A., Kaczmarczyk, P., Wolfeil, N. and Zylicz, A. Labour Mobility within the EU in the context of enlargement and the functioning of the transitional arrangements, Deliverable 6, Centre of Migration Research, University of Warsaw, 2007. Disponível em: $<$ http://publications.wiiw.ac.at/?action= publ\&id = details\&publ= LMS2_6>. Acesso em: 2 de Fevereiro de 2010

FONTES, M. Scientific mobility policies: how Portuguese scientists envisage the return home. Science and Public Policy, v. 34, n. p 284-298, Mai. 2007. 
GAILLARD, A. M. e GAILLARD, J. The International Circulation of Scientists and Technologists - A Win-Lose or Win-Win Situation? Science Communication, v. 20, n.1, p. 106-115, Set. 1998.

GIANNOCOLO, P. OBrain Drain Competition5, ation? e Internatioa Survey.s/d Disponível em: <http:/www.morebrain.net/index htm_files/Brain_Drain_competition__policies_in_Europe.pdf $>$. Acesso em: 15 de Janeiro de 2010.

GIANNOCOLO, P. The Brain Drain. A Survey of the Literature. Working paper, n. 20060302. Univeridade Milão-Bicocca, 2006.

GÓIS, P., MARQUES, J. C. Estudo Prospectivo sobre Imigrantes Qualificados em Portugal, Observatório da Imigração - AltoComissariado para a Imigração e Diálogo Intercultural, Nov. 2007, Lisboa. Disponível em: < http://www.oi.acidi.gov.pt/docs/Estudos\%20 OI/Estudo_OI_24.pdf > . Acesso em: 15 de Janeiro de 2010.

HAKALA, J. Internationalisation of Science - Views of the Scientific Elite. Finland in Science Studies, v. 11, n.1., 1998. Disponível em: $<$ http://www.sciencestudies.fi/system/files/Hakala.pdf $>$. Acesso em: 27 de Fevereiro de 2010

IVATURI, V., LANVIN, B., MOHAN, H. What Will Make People Move, Stay, or Leave in 2015 and Beyond, Global Mobility of Talents. The Global Information Technology Report 2008-2009, 2009.. Disponível em: < http://www.insead.edu/v1/gitr/wef/main/ fullreport/files/Chap1/1.7.pdf> . Acesso em: 26 de Janeiro de 2010

JOHSON, H. G. An "Internationalist" Model. The "brain drain", The Macmillan Company, New York, USA, 1968.

JUBIN, B., Lignères, P. La nouvelle guerre pour les cerveaux, École Nationale Superieure des Mines de Paris, France, 2007.

KURKA, B. International Mobility of highly qualified Austrian professionals in CEE - A literature review on decision making factors, $2^{\text {nd }}$ Central European Conference in Regional Science CERS, Slovakia, 2007. 
Elementos para uma reflexão sociológica sobre o fenômeno da mobilidade...

Sandra Moreira • Emília Rodrigues Araújo

LOWELL, B. L., FINDLAY, A., STEWART, E. (2004) Brain Strain Optimising highly skilled migration from developing countries, Institute for Public Policy Research, UK, Ago.2004. Disponível em: <www.ippr.org/members/download.asp?f=/ecomm/files/ brainstrain.pdf > . Acesso em: 18 de Dezembro de 2009.

MAHROUM, S. Highly skilled globetrotters: mapping the international migration of human capital. R\&D Management, v. 30, n.1, p. 23-32, Jan. 2000.

MARGOLIS, M. "Brain drain". Newsweek, 18 July 2009. Disponível em: <http://www.newsweek.com/id/207382>. Acesso em: 28 de Março de 2010.

MARTINE, George. A Globalização inacabada - Migrações internacionais e pobreza no século 21. São Paulo em perspectiva, v. 19, n. 3, p. 3-22, 2005. Disponível em: < http://www.scielo.br/ pdf/spp/v19n3/v19n3a01.pdf>. Acesso em: 25 de Janeiro de 2010.

MEYER, J. B.; KAPLAN, D.; CHARUM, J. Nomadisme des scientifiques et nouvelle géopolitique des savoirs. Revue Internationale des Sciences Sociales, n. 168, p. 341-354, 2001.

OECD (2002) International Mobility of the Highly Skilled, OECD Policy Brief, July 2002. Disponível em: < http://www.oecd.org/ dataoecd/9/20/1950028.pdf>. Acesso em: 25 de Janeiro de 2010.

PANTINKIN, D. A "Nationalist Model". The "brain drain", Walter Adams (ed.), The Macmillan Company, New York, USA, 1968.

PEIXOTO, J. (2001) Migrações e políticas migratórias na União Europeia: livre circulação e reconhecimento de diplomas. Análise Social, v. 37, p. 153-183, 2001.

PIRRALHA, A., FONTES, M., ASSIS, J.) Assessing Scientific Mobility Dynamics and Impact: drawing on the potential of electronic CV databases. 12th International Conference on Technology, Policy and Innovation (ICTPI),, Porto, Portugal, Jul. 2009. 
RIZVI, F. Rethinking "brain drain" in the Era of Globalisation. Asia Pacific Journal of Education, v. 25, n. 2, p. 175-192, 2005. Disponível em: <http://dx.doi.org/10.1080/02188790500337965>. Acesso em: 10 de Dezembro de 2009.

ROMER, P. M. Economic Growth. 2007. Disponível em: < http:// www.stanford.edu/ promer/EconomicGrowth.pdf > . Acesso em: 9 de Fevereiro de 2010.

SAXENIAN, A. Brain Circulation: How High-Skill Immigration Makes Everyone Better Off. The Brookings Review, v. 20, n. 1, p. 28-31, 2002. Disponível em: < http://people.ischool.berkeley.edu/ anno/ Papers/brain-circulation-brookings-review-2002.pdf > . Acesso em: 14 de Fevereiro de 2010.

SHKOLNIKOV, V. D. Potential Energy - Emergent Emigration of Highly Qualified Manpower from the Former Soviet Union, Rand Graduate School, USA, 1995.

SOLIMANO,A.The International Mobility of Talent:Types, Causes, and Development Impact, Oxford University Press, 2008. Disponível em: $<$ http://books.google.pt/books?id = 8CLH3whiAhsC\&dq = The + Int ernational + mobility + of + talent $\&$ printsec $=$ frontcover\&source $=b l$ \&ots $=$ UevVxlpbxd\&sig $=$ BaakY-jFoOVKkZebBqb7S_AEdAw\&hl =ptPT\&ei $=-14 d S \_y L O M 6 z 4 Q b 1-r H n A g \& s a=X \& o i=$ book_result\&ct $=$ result $\&$ resnum $=5 \& \mathrm{ved}=0 \mathrm{CCUQ} 6 \mathrm{AEwBA \#} \mathrm{v}=$ onepage $\& \mathrm{q}=\& \mathrm{f}=\mathrm{fal}$ se $>$. Acesso em: 2 de Fevereiro de 2010.

SOUBBOTINA, T. Beyond Economic Growth: an introduction to sustainable development, World Bank, Jun.2004. Disponível em: $<$ http://www.worldbank.org/depweb/english/beyond/global/begen.html >. Acesso em: 10 de Fevereiro de 2010.

STARK, O. The New Economics of the "brain drain". World Economics, v. 6, n. 2, Abril-Jun. 2005.

STRAUBHAAR, T. International Mobility of the Highly Skilled: Brain Gain, "fuga de cérebros" or Brain Exchange, HWWA Discussion Paper, Hamburg Institute of International Economics, 


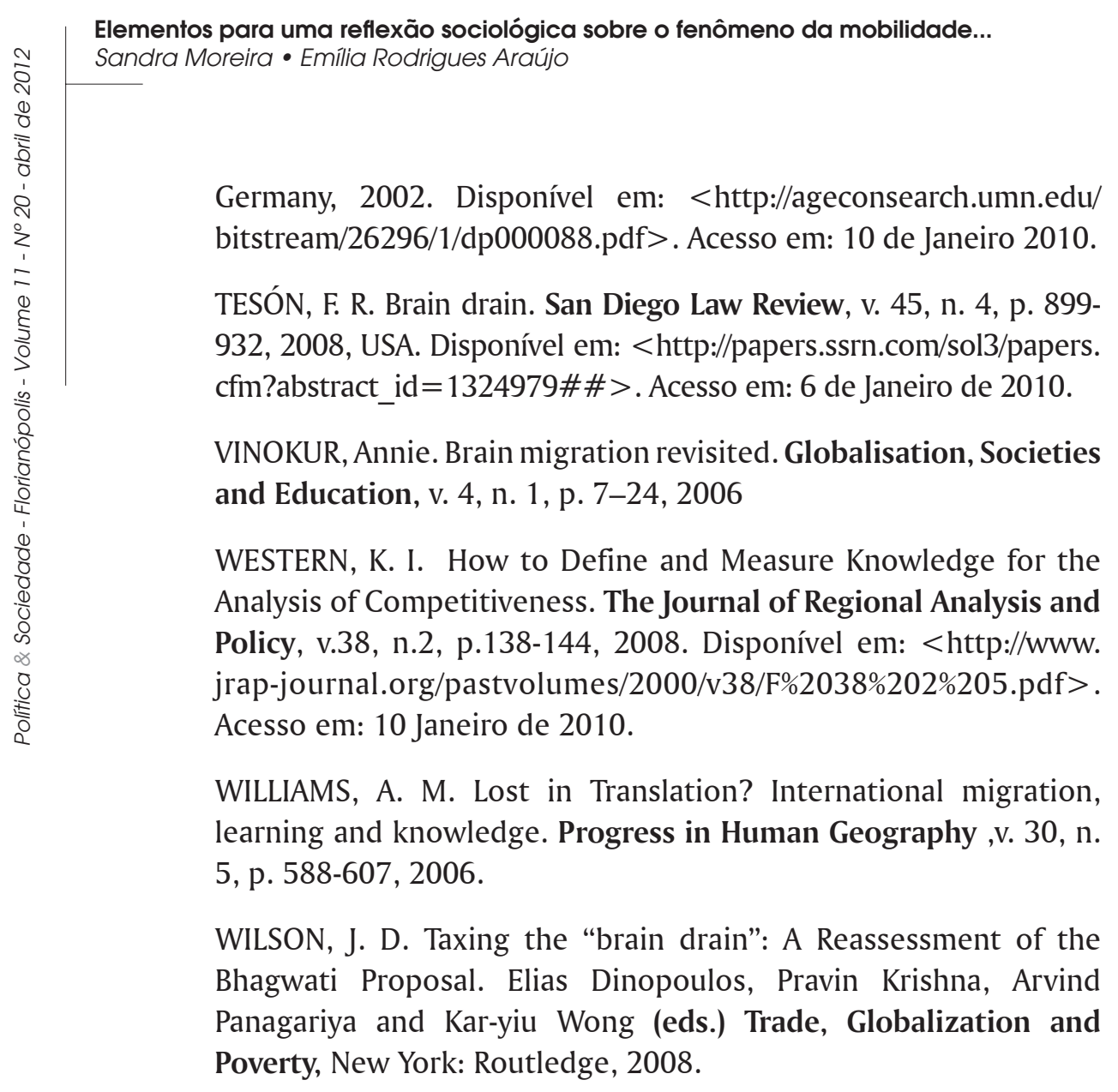

\begin{abstract}
Elements for a sociological reflection on the mobility of researchers and scientists

This paper aims at discussing the concept of mobility of highly skilled professionals and their relationship to the phenomena of brain drain and knowledge circulation. In a context of demographic decline and the consequent increase of mobility, many countries put in place measures to attract new talent that can enhance the dynamics of its institutions and support their economic development. The conclusions to which we arrive emphasize the weakness of the science and technology of small and remote countries such as Portugal, as they constitute attractors of poles of recognized researchers, bringing back the debate about the imbalances between North and South.
\end{abstract}

Keywords: mobility, knowledge circulation. 